\title{
Microstructure and Retention in He-W Co-Deposition Layer
}

\author{
Kosuke ASAI, Naoaki YOSHIDA ${ }^{1)}$, Noriyasu OHNO, Shin KAJITA ${ }^{2)}$, Hirohiko TANAKA, \\ Miyuki YAJIMA ${ }^{3)}$ and Daisuke NAGATA ${ }^{3)}$ \\ Graduate School of Engineering, Nagoya University, Furo-cho, Chikusa-ku, Nagoya 464-8603, Japan \\ ${ }^{1)}$ RIAM, Kyushu University, 6-1 Kasugakoen, Kasuga-shi, Fukuoka 816-8580, Japan \\ ${ }^{2}$ IMaSS, Nagoya University, Nagoya 464-8603, Japan \\ ${ }^{3)}$ National Institute for Fusion Science, Oroshi-cho, Toki, Gifu 509-5292, Japan
}

(Received 9 December 2019 / Accepted 5 January 2020)

\begin{abstract}
A tungsten (W) deposition layer is formed while a sample is exposed to a helium (He) plasma at the surface temperature of 473 or $573 \mathrm{~K}$. The formed $\mathrm{He}-\mathrm{W}$ co-deposition layer was composed of fine grains, the size of which increased with the surface temperature. The samples with the co-deposition layer was exposed to a deuterium $\left(\mathrm{D}_{2}\right)$ plasma, and the $\mathrm{D}$ retention was investigated using thermal desorption spectroscopy. It was found that the co-deposition layer has quite different $\mathrm{D}$ retention characteristics compared with bulk W.
\end{abstract}

(c) 2020 The Japan Society of Plasma Science and Nuclear Fusion Research

Keywords: He-W co-deposition, plasma-material interactions, Co-NAGDIS

DOI: 10.1585/pfr.15.1201004

Tungsten (W) is one of the leading candidates for plasma facing materials (PFM) in fusion reactors. As PFM are exposed to $\mathrm{He}$ in addition to hydrogen isotopes, He atoms are trapped in vacancies and grain boundaries in $\mathrm{W}$, and, consequently, He bubbles cause microstructural changes $[1,2]$. Also, there are regions on PFM where sputtered $\mathrm{W}$ atoms are deposited [3] together with He and hydrogen isotopes. It has been reported that the formation condition of the co-deposition layer, which was formed in a mixture of D and argon gases, strongly change the D retention [4]. Tang et al. demonstrated the D retention of co-deposition layer formed in $\mathrm{He} / \mathrm{D} /$ argon mixture plasmas using a magnetron sputtering device. They revealed that an inclusion of He has great impacts on D retention [5]. Also, Iyyakkunnel et al. investigated co-deposition layer formed in He/argon mixture plasmas using magnetron sputtering device and its He retention [6]. In this study, we focused on He-W co-deposition layer formed under a much higher density condition than magnetron sputtering. Recently, it was found that deposition of $\mathrm{W}$ in He plasma led to a formation of large-scale fiberform nanostructures and porous rough surfaces $[7,8]$ at the surface temperature higher than $\sim 1100 \mathrm{~K}$. In this study, we focused on lower temperature range ( $573 \mathrm{~K}$ or less), considering the condition of the first wall, and investigate the deposition effects.

Figure 1 (a) shows a schematic of the experimental setup of the plasma device Co-NAGDIS. A meander W wire was installed near the sample, and sputtered $\mathrm{W}$ from wire and He plasma deposited simultaneously on a W sample. The sample temperature was controlled by an air cooling system inside the sample stage; the deposition layer was formed at the sample temperature of 473 or $573 \mathrm{~K}$ un-

author'se-mail: asai.kosuke@f.mbox.nagoya-u.ac.jp
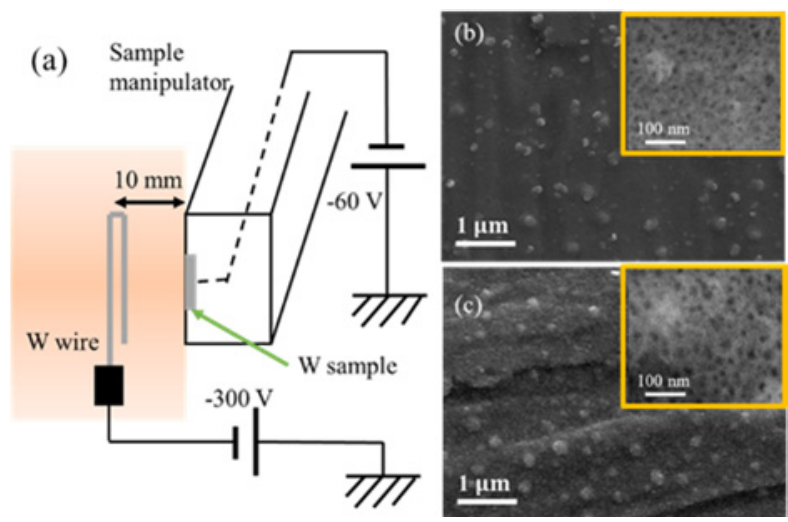

Fig. 1 (a) A schematic view of the experimental setup in the CoNAGDIS device, and (b, c) SEM images of He-W codeposition layer formed at 473 and $573 \mathrm{~K}$, respectively.

der the same plasma condition. Figures $1(b, c)$ show SEM images of the surface of He-W co-deposition layer formed at 473 and $573 \mathrm{~K}$. Especially at $573 \mathrm{~K}$, deposition layer has a several hundred nm blister-like protrusions. Many dots, which can be seen in insets, likely represent He bubbles just below the surface.

Figure 2 shows cross-sectional TEM images of the He-W co-deposited sample. The sample was prepared using focused ion beam (FIB) cutting. At $473 \mathrm{~K}$ (Fig. 2 (a)), high density cavities with diameter of about $1 \mathrm{~nm}$ are seen throughout. Because the cavities became white and black in under focus and over focus cases, respectively, as seen in insets, those should correspond to He bubbles. On the other hand, at $573 \mathrm{~K}$ (Fig. 2 (b)), the bubble size was several tens of nm. Figure 2(c) shows a TEM images of the same sample but a different location, where blister exist, together with an energy dispersive X-Ray spectroscopy 

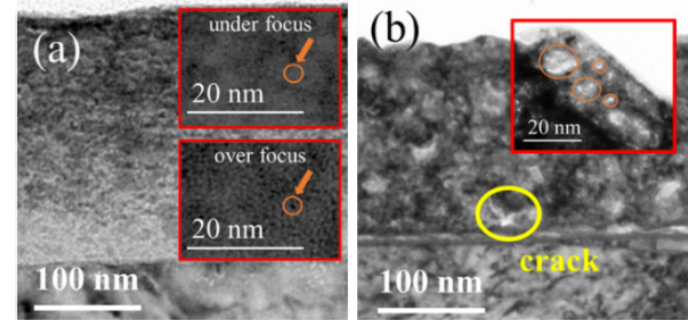

Line analysis area
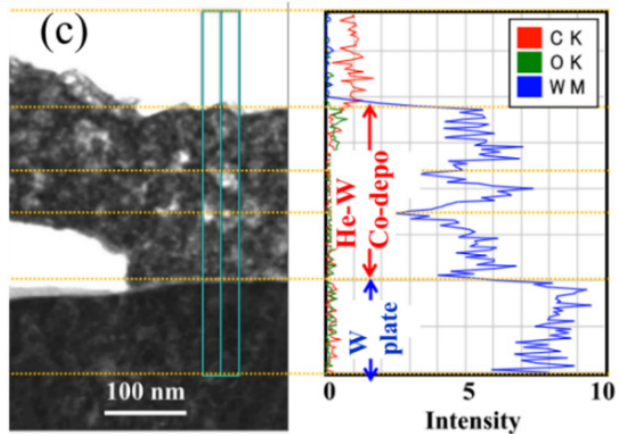

Fig. 2 TEM images of cross-section of He-W co-deposition layer formed at (a) $473 \mathrm{~K}$ and (b) $573 \mathrm{~K}$. (c) TEM image and EDS analysis of $573 \mathrm{~K}$.

(EDS) mapping and an averaged vertical profile of $\mathrm{W}$ signal. The cavity above the substrate suggested that the blister have formed, because the pressure in He bubble near the substrate increased. The EDS analysis clearly shows that the intensity decreased at locations where He bubbles existed, moreover, $\mathrm{W}$ density in co-deposition layer was roughly $62 \%$ of $\mathrm{W}$ substrate. The results indicate that the co-deposition layer is very porous and comprised of fine He bubble and cavities.

After forming He-W co-deposition layer, these samples were exposed to the D plasma. In Fig. 3, desorption amounts of $\mathrm{He}$ and $\mathrm{D}$ were examined by thermal desorption spectroscopy (TDS). As a comparative experiment, bulk $\mathrm{W}$ was also irradiated with the $\mathrm{D}$ plasma. He desorption occurred around $300-900 \mathrm{~K}$. The desorption at $300-$ $600 \mathrm{~K}$ was likely from weak trapping sites such as a heavily distorted lattices probably formed in the co-deposition layers, also the desorption around $900 \mathrm{~K}$ from the sample formed at $573 \mathrm{~K}$ can be caused by the structure changes such as cracks and blisters. Different from desorption of He from bulk W exposed to He plasmas [9-12], where desorption peak appeared around $1000 \mathrm{~K}$, there was no significant desorption around $1000 \mathrm{~K}$ in this study. The reasons can be attributed to the fact that there was no significant amount of He bubbles below the co-deposition layer, as seen in Figs. $2(a, b)$. A He diffusion barrier might have been formed between the deposition layer and $\mathrm{W}$ substrate. Figure 3 (b) shows a comparison of $\mathrm{D}$ desorption between He-W co-deposition layer and bulk W. D retention was much higher than that of bulk $\mathrm{W}$, because of desorption peaks appeared at $300-400 \mathrm{~K}$. It was likely that D atoms were also bound weakly in the co-deposition layer.
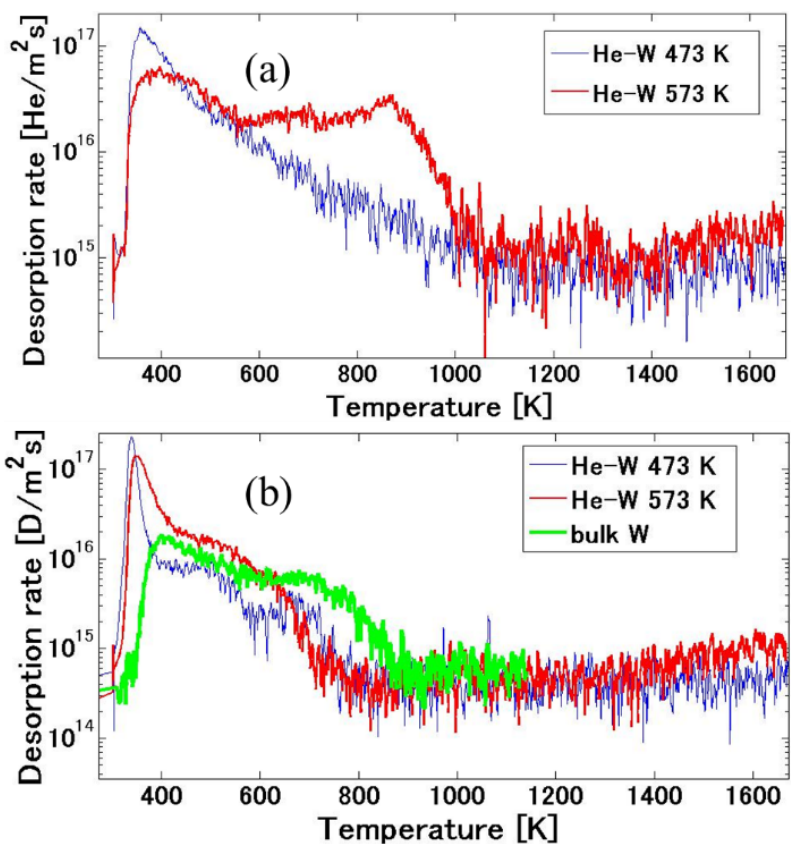

Fig. 3 (a) He and (b) D desorption spectra of He-W codeposition layer after the $\mathrm{D}$ ion irradiation.

In this study, we formed He-W co-deposition layer, and the changes in structures and $\mathrm{He}$ and $\mathrm{D}$ retentions were observed with increasing the temperature. With increasing the surface temperature from 473 to $573 \mathrm{~K}$, the surface roughness was significantly enhanced accompanied by the growth of He bubbles and formation of blisters. He and D desorption occurred at relatively lower temperature than bulk W. To understand the mechanism of the difference in the desorption behaviors, it is of importance to observe structure changes of the co-deposition layer during annealing. The results suggested that co-deposition layer, which can be formed on the first wall of fusion reactors, influences on the particle control.

This work was supported by the National Institute for Fusion Science Collaboration Research Program (NIFS15UFFF039, NIFSULFF016 and NIFS17KNWF003), and by the NINS program of Promoting Research by Networking among Institutions (Grant Number 01411702).

[1] S. Takamura et al., Plasma Fusion Res. 1, 051 (2006).

[2] S. Kajita et al., Nucl. Fusion 49, 095005 (2009).

[3] M. Rubel et al., Fusion Eng. Des. 136, 579 (2018).

[4] G. De Temmerman et al., J. Nucl. Mater. 389, 479 (2009).

[5] X.H. Tang et al., J. Nucl. Mater. 446, 200 (2014).

[6] S. Iyyakkunel et al., ACS Appl. Mater. Interfaces 6, 11609 (2014).

[7] S. Kajita et al., Nucl. Fusion 58, 106002 (2018).

[8] S. Kajita et al., Scientific Reports 8, 9 (2018).

[9] M. Yajima et al., J. Nucl. Mater. 449, 9 (2014).

[10] D. Nishijima et al., J. Nucl. Mater. 337-339, 927 (2005).

[11] K. Tokunaga et al., J. Nucl. Mater. 313-316, 92 (2003).

[12] H.T. Lee et al., J. Nucl. Mater. 360, 196 (2007). 